\title{
Familial partial lipodystrophy type 3 due to PPAR-gamma mutation: presentation with diabetes and severe hypertriglyceridemia

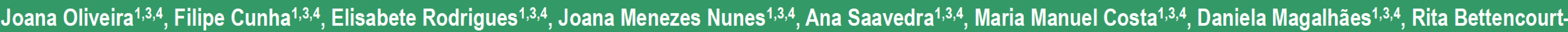
Silva1,3,4, Susana Fernandes ${ }^{2,3}$, João Paulo Oliveira ${ }^{2,3}$, Davide Carvalho ${ }^{1,3,4}$, Paula Freitas ${ }^{1,3,4}$

${ }^{1}$ Endocrinology, Diabetes and Metabolism Department, Centro Hospitalar São João; ${ }^{2}$ Department of Genetics, Centro Hospitalar São João; ${ }^{3}$ Faculty of Medicine, University of Porto; ${ }^{4}$ Institute

for Innovation and Health Research, University of Porto - Porto, Portugal

Introduction: Familial partial lipodystrophy (FPL) is an autosomal dominant disease characterized by selective loss of subcutaneous fat from the extremities and gluteal region, with lipohypertrophy of the face, neck and trunk. It is usually tightly linked with severe metabolic complications. FPL type 3 results from peroxisome proliferator-activated receptor gamma (PPAR-gamma) mutations.



Conclusion

The clinical features and biochemical profile suggested the diagnosis of genetic lipodystrophy, confirmed as FPL type 3 . We underline the importance of clinical suspicion and early intervention of metabolic complications, in order to prevent early onset of cardiovascular disease and the occurrence of pancreatitis.

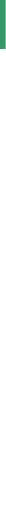

